\title{
Therapeutic effectiveness of rituximab in a patient with unresponsive autoimmune pulmonary alveolar proteinosis
}

\author{
Anat Amital, ${ }^{1}$ Shlomo Dux, ${ }^{2,5}$ David Shitrit, ${ }^{3,5}$ Ofer Shpilberg, ${ }^{4,5}$ \\ Mordechai R Kramer ${ }^{1,5}$
}

${ }^{1}$ Pulmonary Institute Rabin Medical Center Beilinson

Campus, Petah Tiqwa, Israel ${ }^{2}$ Department of Internal Medicine C, Rabin Medical Center Beilinson Campus, Petah Tiqwa, Israel

${ }^{3}$ Pulmonary Department, Meir Medical Center, Kfar Saba, Israel

${ }^{4}$ Department of Hematology, Rabin Medical Center Beilinson Campus, Petah Tiqwa, Israel ${ }^{5}$ Sackler Faculty of Medicine, Tel Aviv University, Tel Aviv, Israel

\section{Correspondence to} Anat Amital, Pulmonary Institute, Rabin Medical Center, Beilinson Campus, Petah Tiqwa 49100, Israel;

aamital@netvision.net.il

Received 16 April 2010 Accepted 5 August 2010

Published Online First

20 September 2010

\begin{abstract}
Pulmonary alveolar proteinosis (PAP) is a rare lung disease characterised by the accumulation of lung surfactant in the alveoli. In most cases it is an autoimmune disease with antibodies directed against the growth factor granulocyte-macrophage colony stimulating factor (GM-CSF). Standard of care consists of whole lung lavages in symptomatic patients. An alternative treatment is GM-CSF injections. The case history is reported of a patient with PAP and severe dyspnoea and hypoxaemia. Whole lung lavages and GMCSF initially resulted in partial remission. However, the patient's condition deteriorated and her saturation during rest with high-flow oxygen treatment was $85 \%$. The patient was treated with an anti-CD20 antibody rituximab which resulted in dramatic improvement. Room air saturation increased to $98 \%$ with exercise and she no longer required supplemental oxygen. The diffusion capacity for carbon monoxide increased from $27 \%$ to $48 \%$ of predicted and the chest $x$-rays improved. Rituximab may be useful in the treatment of patients with unresponsive PAP.
\end{abstract}

\section{INTRODUCTION}

Pulmonary alveolar proteinosis (PAP) is a rare diffuse lung disease first described by Rosen et al in1958. ${ }^{1}$ The condition has multiple aetiologies, including genetic and congenital defects in the surfactant protein B and C genes and in the granulocyte-macrophage colony stimulating factor (GM-CSF)/interleukin 3 (IL-3)/interleukin 5 (IL-5) receptor and secondary to occupational, immunodeficiency and haematological disorders. ${ }^{1}{ }^{2}$ It eventually leads to impaired processing of the surfactant by the alveolar macrophages. Ninety percent of cases are idiopathic and have antibodies directed against GM-CSF. This is thought to result in macrophage dysfunction and impaired immunity. ${ }^{1}$

Rituximab is a monoclonal antibody directed against the CD20 surface antigen on B lymphocytes and has shown substantial benefits for the treatment of some autoimmune disorders mediated by autoantibodies, such as rheumatoid arthritis and idiopathic thrombocytopenia purpura. ${ }^{3}$

\section{CASE REPORT}

A 40-year-old mother of seven who did not smoke developed progressive dyspnoea that had persisted for 6 months. She was diagnosed with PAP in another hospital in 2005 by bronchoalveolar lavage and transbronchial biopsy. There she was treated with three whole lung lavages. When she arrived at our institution she was dyspnoeic at rest, her room air saturation was $84 \%$, requiring 31 of oxygen via nasal cannula. She underwent whole lung lavages in July and November 2005 and her saturation improved to $89-95 \%$ on room air during rest and $87 \%$ during effort; $0.5-11$ oxygen was administered via a nasal cannula.

At the beginning of January 2006, $5 \mathrm{mg} / \mathrm{kg}$ GM-CSF (Leukine; Bayer Healthcare Pharmaceuticals Inc, Leverkusen, Germany) was administered daily. Some improvement (better oxygenation and lung function tests) was demonstrated clinically, but there was no radiographic improvement.

A year later her dyspnoea increased and increasing amounts of supplemental oxygen were required. By February 2007 her saturation was $91 \%$ during rest and $87 \%$ after effort. The dose of GM-CSF was doubled for 2 weeks without improvement (her saturation was 93\% with 61 oxygen via a face mask). By April 2007 saturation on room air was $85 \%$ during rest. The patient again underwent two whole lung lavages which were difficult to perform owing to severe hypoxaemia. During the first procedure (April 2007) both lungs were lavaged. At the end of the procedure she required cardiopulmonary resuscitation because of hypoxaemia (P/F ratio $83 \mathrm{~mm} \mathrm{Hg}$ ), bradycardia (30 beats/min) and hypotension. High fever, leucocytosis and pneumonia with sepsis followed. She was admitted to the ICU requiring mechanical intubation for 3 days. During the second procedure (September 2007) hypoxaemia occurred earlier (P/F ratio $61 \mathrm{~mm} \mathrm{Hg}$ ) and carbon dioxide tension was $67 \mathrm{~mm} \mathrm{Hg}$, so only the left lung was lavaged with 12 litres. Chest x-rays demonstrated right pneumothorax and pneumomediastinum and she was transferred to the ICU. After resolution of the barotrauma, the patient remained hypoxaemic despite high oxygen requirements.

The anti-GM-CSF antibody titre measured in November 2007 was >1:12800 (normal <1:400) (Brody School of Medicine Laboratories at East Carolina University, North Carolina, USA). A course of $375 \mathrm{mg} / \mathrm{m}^{2}$ rituximab was administered weekly over 4 weeks. There were no adverse effects and the improvement was dramatic. Oxygen therapy was stopped after 4 weeks, lung carbon monoxide diffusion capacity (DLCO) improved and oxygen saturation increased to $94 \%$ on room air The lactate dehydrogenase level decreased from 1062 U/l in November 2007 to 589 U/l. The patient 


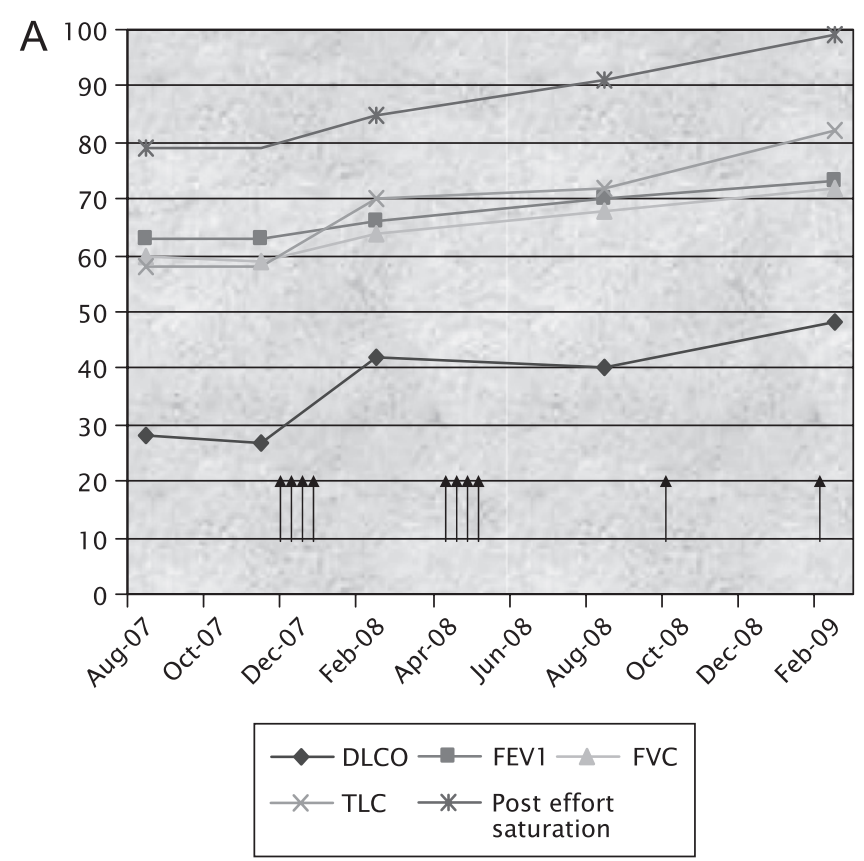

B

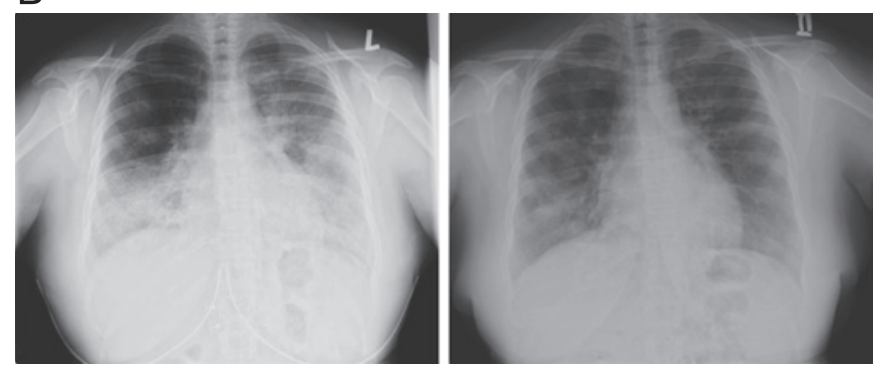

Figure 1 (A) Treatment with rituximab improved lung function. Four arrows together represent a 4-week course and single arrows represent maintenance treatment with a single infusion course. (B) Chest $x$-rays before and after treatment. Dıco, lung carbon monoxide diffusion capacity; FVC, forced vital capacity; FEV 1 , forced expiratory volume in $1 \mathrm{~s}$; TLC, total lung capacity.

still had effort dyspnoea. After a second course of rituximab her Dico and oxygen saturation at rest and during exercise further improved. She was able to walk $420 \mathrm{~m}$ in $6 \mathrm{~min}$ compared with $198 \mathrm{~m}$ in August 2007 before treatment. Following a single maintenance infusion of rituximab, saturation reached $98 \%$ during effort. Dico was $48 \%$ of predicted and the chest x-rays and CT scan improved (figure 1), despite the fact that the antiGM-CSF antibody titre remained elevated (1:12 000). Although the patient is not completely well, she is living a full life at home and participating in physical activities without any need for supplemental oxygen.

\section{DISCUSSION}

This patient had severe PAP which responded to treatment with rituximab after failure of other therapies. The patient improved clinically and physiologically, her room air saturation increased to $98 \%$ with exercise, she no longer required supplemental oxygen, Dico increased from $27 \%$ to $48 \%$ of predicted and she could walk $420 \mathrm{~m}$ in 6 min compared with $198 \mathrm{~m}$ before treatment.

The standard treatment for patients with PAP is whole lung lavage, but this treatment has serious disadvantages and side effects. There is a need for general anaesthesia, mechanical ventilation of one lung in a hypoxaemic patient may be difficult and leakage of lavage fluid to the ventilated lung may occur. Pneumothorax, pneumomediastinum and infections occur infrequently. Another treatment is GM-CSF which is effective in $50-70 \%$ of patients but its effect may diminish. ${ }^{1}$

Idiopathic PAP is an autoimmune disease. Immunosuppressive agents may have a negative effect on macrophage function and may even cause secondary PAP. We chose a therapy that would least affect the number and function of macrophages. In this case, the GM-CSF antibody titre was not affected. A review of the literature found a single case report where rituximab was used in a patient who refused lavages. In that case the GM-CSF titre decreased after 3 months. ${ }^{4}$ The titre also decreased after treatment with plasmapheresis. ${ }^{5}$ There is no correlation between GM-CSF titre and severity of lung disease, and there is conflicting evidence concerning the level of GM-CSF antibodies and response to treatment. ${ }^{2}{ }^{4}$ Because our patient had a very high titre, it is possible that it would take longer to see an improvement in the titres.

PAP may resolve spontaneously. However, considering the previous course of her disease, we think it is unlikely that our patient had a spontaneous remission and the time to improvement was similar to that for other conditions treated with this agent.

We conclude that rituximab should be considered as an optional treatment for PAP in patients who do not respond to whole lung lavage or antibody therapy. ${ }^{6}$

\section{Competing interests None.}

Patient consent Obtained.

Ethics approval Treatment was approved by the hospital drug approval committee.

Provenance and peer review Not commissioned; externally peer reviewed.

\section{REFERENCES}

1. Ioachimescu OC, Kavuru MS. Pulmonary alveolar proteinosis. Chron Respir Dis 2006;3:149-59.

2. Inoue $\mathbf{Y}$, Trapnell BC, Tazawa R, et al. Japanese Center of the Rare Lung Diseases Consortium. Characteristics of a large cohort of patients with autoimmune pulmonary alveolar proteinosis in Japan. Am J Respir Crit Care Med 2008;177:752-62.

3. Lim SH, Beers SA, French RR, et al. Anti-CD20 monoclonal antibodies: historical and future perspectives. Haematologica 2010:95:135-43.

4. Borie R, Debray MP, Laine C, et al. Rituximab therapy in autoimmune pulmonary alveolar proteinosis. Eur Respir J 2009;33:1503-6.

5. Luisetti M, Rodi G, Perotti C, et al. Plasmapheresis for treatment of pulmonary alveolar proteinosis. Eur Respir J 2009;33:1220-2.

6. Colombat $\mathbf{P}$, Salles G, Brousse N, et al. Rituximab (anti-CD20 monoclonal antibody) as single first-line therapy for patients with follicular lymphoma with a low tumor burden: clinical and molecular evaluation. Blood 2001;97:101-6.

7. Garcia-Chavez J, Majuf-Cruz A, Montiel-Cervantes L, et al. Mexican Hematology Study Group. Rituximab therapy for chronic and refractory immune thrombocytopenic purpura: a long-term follow-up analysis. Ann Hematol 2007;86:871-7. 